\title{
COMPONENTS OF VARIATION UNDER SIB-MATING
}

\author{
B. I. HAYMAN \\ Agricultural Research Council's Unit of Biometrical Genetics, \\ Department of Genetics, University of Birmingham
}

Received 9.iv.52

\section{INTRODUCTION}

MAther (1949a and $b$ ) has described how the heritable portion of continuous variation in certain experimental populations may be partitioned into two components, $D$ and $H$, the former depending upon differences in phenotype between homozygotes, and the latter upon departures in phenotype of heterozygotes from the average expression of corresponding homozygotes. Bateman and Mather (I95I) considered the case of selfing after an initial cross of two inbred lines and obtained (as powers of $\frac{1}{2}$ ) the general formulx for the coefficients of $D$ and $H$ in the different measures of variation in each generation. Nelder (1952) has described a general method of obtaining these coefficients for various systems of inbreeding and of obtaining the forms of $D$ and $H$ when linkage causes these to vary from generation to generation.

We have used Nelder's method to obtain the coefficients of $D$ and $H$ under sib-mating after an initial cross of two inbred lines. These coefficients are, of course, essential for the analysis of inbreeding experiments using sib-mating, whether as in animals as the closest possible system of inbreeding, or in plants as a system intermediate between selfing and random mating.

\section{COMPONENTS OF VARIATION}

Consider two alleles, $A$ and $a$, of one gene, and the possible genotypes $A A, a a, A a$. The possible mating pairs are given on the left of table $I$, and their proportions in a population form a 6-vector which under sib-mating changes according to the transformation matrix,

$$
\begin{array}{l|cccccc|}
A A \times A A & \mathrm{I} & \cdot & \frac{1}{4} & . & \frac{1}{16} & . \\
a a \times a a & \cdot & \mathrm{I} & \cdot & \frac{1}{4} & \frac{1}{16} & . \\
A A \times A a & \cdot & \cdot & \frac{1}{2} & . & \frac{1}{4} & . \\
a a \times A a & \cdot & \cdot & \cdot & \frac{1}{2} & \frac{1}{4} & . \\
A a \times A a & \cdot & \cdot & \frac{1}{4} & \frac{1}{4} & \frac{1}{4} & \mathrm{I} \\
A A \times a a & \cdot & \cdot & \cdot & \cdot & \frac{1}{8} & .
\end{array}
$$

Denote by $S_{0}$ the generation in which the homozygotes $A A$ and $a a$ are crossed, and by $S_{l}$ the $l$ th generation of subsequent sib-mating. The first two generations $S_{1}$ and $S_{2}$ coincide of course with the $F_{1}$ and $\mathrm{F}_{2}$ of a selfing series. The proportions of mating pairs in $S_{l}$ may be 
obtained from the latent roots and vectors of the above matrix and are given in table $\mathrm{I}$.

TABLE I

$\begin{aligned} A A & \times A A \\ a & \times a a \\ A A & \times A a \\ a & \times A a \\ A a & \times A a \\ A A & \times a a\end{aligned}$

$$
\begin{array}{c|c} 
& \frac{5}{2}-4 g(l+4)+4^{-l} \\
1 . & \frac{5}{2}-4 g(l+4)+4^{-l} \\
5 & g(l)-4^{-l+1} \\
& g(l)-4^{-l+1} \\
& g(l-1)+4^{-l+1} \\
& \frac{1}{x} g(l-2)+2 \cdot 4^{-l}
\end{array}
$$

where $g(l)=2^{-l+1}(2 f(l+I)-f(l))$ and $f(l)$ is the Fibonacci series $\mathrm{o}, \mathrm{I}, \mathrm{I}, 2,3,5,8, \mathrm{I} 3, \ldots$ with $f(\mathrm{o})=\mathrm{o}, f(\mathrm{I})=\mathrm{I}, f(l+2)=f(l+\mathrm{I})+$ $f(l)$ and $f(-l)=(-)^{l-1} f(l)$.

Following Nelder, we say that plants of generation $S_{k}$, which have a common ancestral pair in $S_{l}(l<k)$ but not in $S_{l+1}$, form an $l$-group. We define $V_{l s k}$ as that item in the heritable variance of generation $S_{k}$ which is given by the variance of the means of $(k-l+\mathrm{I})$-groups taken round the means of the $(k-l)$-groups and averaged over the latter. Hence $V_{l S k}$ can be expressed as the difference of the total variance of $(k-l+\mathrm{I})$-group means and $(k-l)$-group means in $S_{k}$. We compute the variance of $(k-l)$-group means using the mean of $S_{k-l}$ as variate while the frequencies are those of $S_{l}$.

Let $d,-d$ and $h$ be the deviations of the phenotypes of $A A$, aa and $A a$ respectively from the mean pheurulype of $A A$ and $a a$. Then the contributions of the various mating pairs to the mean of $S_{k-l}$ are given in table 2.

$$
\mid \begin{gathered}
\text { TABLE 2 } \\
d \\
-d \\
\frac{1}{2} d+2^{-k+l-1} f(k-l+2) h \\
-\frac{1}{2} d+2^{-k+l-1} f(k-l+2) h \\
2^{-k+l} f(k-l+1) h \\
2^{-k+l+1} f(k-l) h
\end{gathered}
$$

Writing $D$ for $d^{2}$ and $H$ for $h^{2}$ we find after some reduction that

$$
\begin{aligned}
& V_{l S k}=2^{-l-1} f(l) D+2^{-2 k+1}\left\{4(-)^{k-l}\right.+2^{l}(-)^{k-l}(3 f(l-2)-f(l-1)) \\
&+2^{l-1}(27 f(2 k-l-2)-4 f(2 k-l-1) \\
&-f(2 k-2 l-3)-8 f(2 k-2 l-2) \\
&\left.+(-2)^{l-1}(f(2 k-3 l-5)+8 f(2 k-3 l-4))\right\} H / 25 .
\end{aligned}
$$

We must make one correction. Table 2 is incorrect for $k-l=1$, since in this case it gives the mean of each mating pair, while for $V_{(k-1) S k}$ we want the variance of individuals and not of pairs about their family means. It happens, as we might therefore expect, that the above formula for $V_{l S k}$ must be doubled when $l=k-\mathbf{I}$. Tables 3 and 4 contain the coefficients of $D$ and $H$ in $V_{l S k}$ up to $k=$ io.

The formula for the coefficient of $H$ is cumbersome, but we need only use it to calculate the nine results for $l=\mathrm{I}, 2,3$ and $k-l=\mathrm{I}, 2,3$. 
Denote the coefficient of $2^{-2 k+1} H$ in $V_{l S k}$ by $h(l, k)$. These are the figures in the main body of table 4 (but multiplied by 2 in the leading diagonal). Then

and

$$
h(l, k+3)=2 h(l, k+2)+2 h(l, k+\mathrm{I})-h(l, k)
$$

$$
h(l+3, k+3)=3^{h}(l+2, k+2)+2 h(l+\mathrm{I}, k+\mathrm{I})-4 h(l, k) .
$$

These enable the remainder of the table to be constructed by extension along the rows and diagonals. As examples of the use of the tables we note that

$$
V_{2 S 4}=\frac{\mathrm{I}}{8} D+\frac{5}{\mathrm{I} 28} H \quad(\text { directly from tables } 3 \text { and } 4)
$$

and

$$
V_{4 S 11}=\frac{3}{3^{2}} D+\frac{64 \mathrm{II}}{2097 \mathrm{I}^{2}} H \text { (by extension from table } 4 \text { since }
$$

$64 \mathrm{II}=2 \times 245^{\circ}+2 \times 935-359$ and 2097 I $\left.52=4 \times 524288\right)$.

For several genes segregating independently, $D$ and $H$ become $\Sigma d^{2}$ and $\Sigma h^{2}$. With linkage the forms of $D$ and $H$ change from

\begin{tabular}{|c|c|c|c|c|c|c|c|c|c|}
\hline & 2 & 3 & 4 & 5 & 6 & 7 & 8 & 9 & 10 \\
\hline $\begin{array}{l}1 \\
2 \\
3 \\
4 \\
5 \\
6 \\
7 \\
8 \\
9\end{array}$ & 2 & $\begin{array}{l}2 \\
6\end{array}$ & $\begin{array}{r}3 \\
5 \\
22\end{array}$ & $\begin{array}{r}9 \\
12 \\
19 \\
70\end{array}$ & $\begin{array}{r}22 \\
31 \\
42 \\
59 \\
230\end{array}$ & $\begin{array}{r}59 \\
81 \\
111 \\
138 \\
195 \\
742\end{array}$ & $\begin{array}{r}153 \\
212 \\
287 \\
359 \\
450 \\
627 \\
2406\end{array}$ & $\begin{array}{r}402 \\
555 \\
754 \\
935 \\
1175 \\
1458 \\
2035 \\
7782\end{array}$ & $\begin{array}{r}1051 \\
1453 \\
1971 \\
2450 \\
3055 \\
3799 \\
4722 \\
6579 \\
25190\end{array}$ \\
\hline divisors & 8 & 32 & 128 & $5^{12}$ & 2048 & 8192 & 32768 & 131072 & 524288 \\
\hline
\end{tabular}

TABLE 3

\begin{tabular}{|r|r|r|r|r|r|r|r|r|r|r|r|}
\hline$l$ & $k$ & 2 & 3 & 4 & 5 & 6 & 7 & 8 & & \\
\hline \\
\hline
\end{tabular}

TABLE 4 
generation to generation, but in all cases the coefficients of $D$ and $H$ in $V_{I S k}$ remain unaltered.

It should be noted that a law of conservation of heritable variation holds (Mather, 1949b). The grand mean of $S_{k}$ is $2^{-k+1} f(k) h$. The square of the coefficient of $h$ plus the sum of the coefficients of $D$ and $H$, for given $k$, is always unity.

\section{COVARIANCES}

Let $W_{l S(k-1) k}$ be the covariance between $(k-l)$-group means in $S_{k}$ and $S_{k-1}$. Instead of using the squares of terms in table 2 with the frequencies of table $I$ we construct an auxiliary table from table 2 by replacing $k$ by $k-1$ and then use the products of corresponding terms from table 2 and the auxiliary table with the frequencies of table I. Hence

$$
\begin{aligned}
W_{l S(k-1) k}= & 2^{-l-1} f(l) D+2^{-2 k+2}\left\{2(-)^{k-l-1}\right. \\
& +2^{l-1}(-)^{k-l}(f(l-1)-3 f(l-2)) \\
& +2^{l-1}(27 f(k-l-3)-4 f(2 k-l-2)) \\
& -f(2 k-2 l-4)-8 f(2 k-2 l-3) \\
& \left.+(-2)^{l-1}(f(2 k-3 l-6)+8 f(2 k-3 l-5))\right\} H / 25 .
\end{aligned}
$$

The coefficients of $D$ are independent of $k$ and are the same as for $V_{l S k}$. Table 5 contains the coefficients of $H$. Once the first nine terms have been evaluated the table may be extended using the same relations as for the variance table. Since $k-l>\mathbf{I}$ a correction like that in the previous section is not needed.

Examples $\quad W_{1 S 23}=\frac{1}{4} D$

(Tables 3 and 5.)

$$
W_{4 S 87}=\frac{3}{3^{2}} D+\frac{81}{4096} H
$$

\begin{tabular}{|c|c|c|c|c|c|c|c|c|}
\hline 1 & 3 & 4 & 5 & 6 & 7 & 8 & 9 & 10 \\
\hline $\begin{array}{l}1 \\
2 \\
3 \\
4 \\
5 \\
6 \\
7 \\
8\end{array}$ & o & $\begin{array}{l}2 \\
2\end{array}$ & $\begin{array}{l}5 \\
7 \\
6\end{array}$ & $\begin{array}{l}14 \\
19 \\
25 \\
22\end{array}$ & $\begin{array}{l}36 \\
50 \\
67 \\
81 \\
70\end{array}$ & $\begin{array}{r}95 \\
131 \\
138 \\
219 \\
219 \\
230\end{array}$ & $\begin{array}{l}248 \\
343 \\
465 \\
578 \\
775 \\
857 \\
742\end{array}$ & $\begin{array}{r}650 \\
898 \\
1219 \\
1513 \\
1890 \\
2315 \\
2777 \\
2406\end{array}$ \\
\hline divisors & 16 & 64 & 256 & 1024 & 4096 & $16_{3} 8_{4}$ & $6_{5536}$ & 262144 \\
\hline
\end{tabular}

Covariances between means $m$ generations apart may be denoted by $W_{l S(k-m) k}$. The coefficients of $D$ are the same as before, and

TABLE 5 
the coefficients of $H$ may be obtained simply from tables 4 and 5 as follows. Let $h(l, k, m)$ be the coefficient of $2^{-2 k+m+1} H$ in $W_{l S(k-m) k}$. Then $h(l, k, m+2)=-h(l, k, m+1)+h(l, k, m)$. This means for example that the main body of the $W_{l S(k-2) k}$ table is the difference of the main bodies of tables 4 and 5 , and the divisors in the $W_{l S(k-2) k}$ table are half those in table 5 .

$$
\begin{aligned}
& \text { Examples } \quad V_{3 S 7}=\frac{2}{\mathrm{I} 6} D+\frac{\mathrm{II} \mathrm{I}}{8 \mathrm{I} 9^{2}} H \quad \text { (Tables } 3 \text { and } 4 \text {.) } \\
& W_{3 S 67}=\frac{2}{16} D+\frac{67}{4096} H \quad(\text { Tables } 3 \text { and 5.) } \\
& W_{3 S 57}=\frac{2}{16} D+\frac{44}{204^{8}} H \quad(\text { since } 44=\mathrm{III}-67) . \\
& W_{3547}=\frac{2}{16} D+\frac{23}{1024} H \quad(\text { since } 23=67-44) .
\end{aligned}
$$

\section{SUMMARY}

The coefficients of $D$ and $H$ in the different measures of continuous variation are obtained for the generations of a population started by a cross between true breeding lines and maintained by continued sib-mating. The coefficients are set out for generations up to $S_{10}$ in tables, 3,4 and 5 .

\section{REFERENCES}

MATHER, K. 1949a. Biometrical Genetics. Methuen \& Co. Ltd., London.

MATHER, K. I $949 b$. The genetical theory of continuous variation. Hereditas, Suppl. Vol., pp. 376-401.

Bateman, A. J., AND MATHER, K. 1951. The progress of inbreeding in barley. Heredity, 5,324 .

NELDER, J. A. 1952. Some genotypic frequencies and variance components occurring in biometrical genetics. Heredity, 6, 387-394. 